\title{
EXACT MULTIPLICITY FOR PERIODIC SOLUTIONS OF DUFFING TYPE
}

\author{
Hongbin Chen ${ }^{\mathrm{a}}$, Yi Li ${ }^{\mathrm{b}, *}$, Xiaojie $\mathrm{Hou}^{\mathrm{b}}$ \\ a Department of Mathematics, Xi'an Jiaotong University, Xi'an, 710049 P.R. China \\ ${ }^{\mathrm{b}}$ Department of Mathematics, University of Iowa, Iowa City, Iowa 52242
}

\begin{abstract}
In this paper, we study the following Duffing-type equation:

$$
x^{\prime \prime}+c x^{\prime}+g(t, x)=h(t)
$$

where $g(t, x)$ is a $2 \pi$-periodic continuous function in $t$ and concave-convex in $x$, and $h(t)$ is a small continuous $2 \pi$-periodic function. The exact multiplicity and stability of periodic solutions are obtained.
\end{abstract}

Key Words: Periodic Solution; Topological Degree; Stability Index.

2000 Mathematics Subject Classification: 34c25, 34c10.

\section{INTRODUCTION}

This paper is devoted to the exact multiplicity of periodic solutions of the perturbation Duffing-type equation

$$
x^{\prime \prime}+c x^{\prime}+g(t, x)=h(t)
$$

where $g(t, x)$ is a continuous $2 \pi$-periodic function in $t$.

\footnotetext{
${ }^{*}$ Corresponding author; Fax: (319)-335-0627

E-mail address: yi-li@uiowa.edu (Yi Li).
} 
The existence and multiplicity of periodic solutions of (1.1) or more general types of nonlinear equations of second order has been investigated extensively by many authors since C. Fabry and J.M. Nkashama initiated the study of the AmbrosettiProdi problem with periodic boundary conditions $[4,6,9,10]$. The question of sharp estimates of the number of solutions is less extensively studied $[5,7,8]$, R. Ortega studied (1.1) from the stability point of view and obtained an Ambrosetti-Proditype [1] theorem under convex nonlinearity. The aim of this paper is to understand the effects of concave-convex nonlinearities on the set structure of solutions of (1.1). Our results have been motivated by Castro and Lazer's [2] concerning the number of solutions of the Dirichlet problem for partial differential equations, but their method, which is based on a variational technique, cannot apply to (1.1), since linearization of the periodic problem (1.1) is a non-self-adjoint operator. We obtain multiplicity for periodic solutions by means of a topological degree combined with a Sturm comparison argument, and stability of periodic solutions follows by computing a local index developed by R. Ortega [8].

Now we state our main results.

Theorem 1. Assume that the function $g(t, x)$ in equation (1.1) is $C^{2}$ and $2 \pi-$ periodic in $t$, such that the following conditions are satisfied:

1. $g(t, 0)=0, g^{\prime}(t, 0) \ll 0$ and $g^{\prime \prime}(t, x) x>0$ for $x \neq 0$;

2. $\lim _{x \rightarrow-\infty} g^{\prime}(t, x)=\alpha(t)$ and $\lim _{x \rightarrow \infty} g^{\prime}(t, x)=\beta(t)$ uniformly in $t \in \mathbb{R}$;

3. $0 \ll \alpha(t), \beta(t) \ll 1+\frac{c^{2}}{4}$.

Then there is $r>0$ such that for $h(t) \in C(\mathbb{R} / 2 \pi \mathbb{Z})$ and $\|h(t)\| \leq r$ the differential equation (1.1) has exactly three $2 \pi$-periodic solutions. Furthermore, two periodic solutions are asymptotically stable and one of them with small magnitude is unstable if $\alpha(t), \beta(t) \ll \frac{1}{4}\left(1+c^{2}\right)$ and $c>0$.

Similarly we have the following theorem. 
Theorem 2. Assume that the following conditions hold:

1. $g(t, x) \in C^{2}(\mathbb{R} \times \mathbb{R} / 2 \pi \mathbb{Z})$ such that $g(t, 0) \equiv 0$ and $0 \ll g^{\prime}(t, 0) \ll 1+c^{2} / 4$;

2. $\lim _{x \rightarrow-\infty} g^{\prime}(t, x)=\alpha(t)$ and $\lim _{x \rightarrow \infty} g^{\prime}(t, x)=\beta(t)$ uniformly in $t \in \mathbb{R}$;

3. $p(t)=\max \{\alpha(t), \beta(t)\} \ll 0$.

Then there is $r>0$, such that for all $h(t) \in C(\mathbb{R} / 2 \pi \mathbb{Z})$ and $\|h(t)\|<r$, equation (1.1) has exactly three periodic solutions. Furthermore one of the periodic solutions is asymptotically stable and the other two are unstable if $g(t, 0) \ll \frac{1}{4}\left(1+c^{2}\right)$.

Next we consider the Liénard equation

$$
x^{\prime \prime}+f(x) x^{\prime}+g(x)=h(t)
$$

where $f$ and $g \in C^{1}(\mathbb{R})$. We have the following uniqueness result.

Theorem 3. Suppose that $g^{\prime}(x)<0$. Then the Liénard equation has a unique $2 \pi$-periodic solution if and only if $\bar{h} \in g(\mathbb{R})$, where $\bar{h}=\frac{1}{2 \pi} \int_{0}^{2 \pi} h(t) d t$.

The following notations are used.

$L_{2 \pi}^{p} \quad 2 \pi$-periodic function $u \in L^{p}[0,2 \pi]$ with $\|u\|_{p}$ for $1 \leq p \leq \infty$;

$C_{2 \pi}^{k} \quad 2 \pi$-periodic function $u \in C^{k}[0,2 \pi], k \geq 0$, with $C^{k}$-norm.

\section{TOPOLOGICAL INDEX AND LINEAR PERIODIC PROBLEM}

2.1. Continuation theorem and index. In this subsection we shall recall some basic results about the topological method. Consider the periodic boundary value problem

$$
\begin{aligned}
x^{\prime} & =f(t, x), \\
x(0) & =x(2 \pi),
\end{aligned}
$$

where $f:[0,2 \pi] \times \mathbb{R}^{n} \rightarrow \mathbb{R}^{n}$ is continuous and $2 \pi$-periodic in $t$. In order to use homotopic methods to compute the degree, we assume that $h:[0,2 \pi] \times \mathbb{R}^{n} \times[0,1] \rightarrow$ 
$\mathbb{R}^{n}$ is a continuous function such that

$$
\begin{aligned}
& h(t, x, 1)=f(t, x), \\
& h(t, x, 0)=g(x),
\end{aligned}
$$

where $g(x)$ is continuous. The following continuation theorem is due to J. Mawhin $[7]$.

Lemma 2.1. Let $\Omega \subset C_{2 \pi}$ be an open bounded set such that the following conditions are satisfied:

(1) There is no $x \in \partial \Omega$ such that

$$
x^{\prime}=h(t, x, \lambda) \quad \forall \lambda \in[0,1)
$$

(2) $\operatorname{deg}\left(g, \Omega \cap \mathbb{R}^{n}, 0\right) \neq 0$.

Then (2.1)-(2.2) has at least one solution.

Let us consider the following Liénard equation:

$$
x^{\prime \prime}+f(x) x^{\prime}+g(t, x)=h(t)
$$

where $h(t) \in C_{2 \pi}$. Evidently the periodic solution of (2.3) is equivalent to the planar system

$$
\begin{aligned}
& x^{\prime}=y-F(x) \\
& y^{\prime}=h(t)-g(t, x)
\end{aligned}
$$

where $F(x)$ is a primitive of the $f(x)$. A natural choice for the homotopy in applying Lemma 2.1 is to take

$$
h(t, x, y, \lambda)=\{y-F(x), \lambda h(t)+(1-\lambda) \bar{h}-[\lambda g(t, x)+(1-\lambda) \bar{g}(x)]\}
$$


where $\bar{g}(x)=\frac{1}{2 \pi} \int_{0}^{2 \pi} g(t, x) d t$ is the average of $g(t, x)$. Since $h(t, x, 0)=(y-$ $F(x), \bar{p}-\overline{g(x)})=G(x)$, then the condition (2) in Lemma 2.1 reduces to

$$
\operatorname{deg}\left(G, \Omega \cap \mathbb{R}^{n}, 0\right) \neq 0 .
$$

Next we consider system (2.1) for $n=2$. We denote by $x\left(t, x_{0}\right)$ the initial value solution of (2.1) and introduce the Poincaré map $P: x_{0} \rightarrow x\left(2 \pi, x_{0}\right)$. It is well known that $x\left(t, x_{0}\right)$ is a $2 \pi$-periodic solution of system (2.1) if and only if $x_{0}$ is a fixed point of $P$. If $x$ is an isolated $2 \pi$-periodic solution of $(2.1)$, then $x_{0}$ is an isolated fixed point of $P$. Hence the Brouwer index is defined by

$$
\operatorname{ind}\left(x_{0}\right)=\operatorname{ind}\left[P, x_{0}\right]=\operatorname{deg}\left(I-P, B_{\varepsilon}(0), 0\right) .
$$

Definition. A $2 \pi$-periodic solution $x$ of (2.1) will be called a nondegenerate $2 \pi$ periodic solution if the linearized equation

$$
y^{\prime}=f_{x}(t, x) y
$$

does not admit a nontrivial $2 \pi$-periodic solution.

Let $M(t)$ be the fundamental matrix of (2.7), and $\mu_{1}$ and $\mu_{2}$ be eigenvalues of the matrix $M(2 \pi)$. Then $x\left(t, x_{0}\right)$ is asymptotically stable if and only if $\left|\mu_{i}\right|<1$, $i=1,2$, otherwise $x\left(t, x_{0}\right)$ is unstable.

The following connection between stability and topological index is due to R. Ortega $[8]$.

Lemma 2.2. Assume that $x$ is an isolated $2 \pi$-periodic solution of (1.1) such that the condition

$$
g_{x}(t, x) \leq \frac{1}{4}\left(1+c^{2}\right)
$$

holds, for $t \in \mathbb{R}, c>0$. Then $x$ is asymptotically stable (resp., unstable) if and only if $\operatorname{ind}(x)=1$ (resp., ind $(x)=-1)$. 
2.2. Linear periodic equation. Consider the homogeneous periodic equation

$$
L_{\alpha} x=x^{\prime \prime}+c x^{\prime}+\alpha(t) x=0
$$

where $c$ is a constant and $\alpha(t) \in L_{2 \pi}$.

The following simple lemma is the key to proving our main results.

Lemma 2.3. Suppose that $\alpha(t), \alpha_{1}(t)$ and $\alpha_{2}(t) \in L_{2 \pi}$ such that

$$
\alpha_{1}(t), \alpha_{2}(t) \text { and } \alpha(t) \ll 1+\frac{c^{2}}{4} \text {. }
$$

Then

(1) The possible $2 \pi$-periodic solution $x$ of equation (2.9) is either trivial or different from zero for each $t \in \mathbb{R}$.

(2) $L_{\alpha_{i}} x=0(i=1,2)$ cannot admit nontrivial $2 \pi$-periodic solutions simultaneously if $\alpha_{1}(t) \ll \alpha_{2}(t)$.

(3) $L_{\alpha} x=0$ has no nontrivial $2 \pi$-periodic solution, and $\operatorname{ind}\left(L_{\alpha}, 0\right):=\operatorname{deg}\left(L_{\alpha}, B_{\varepsilon}, 0\right)=$ 1 (resp., $=-1)$ if $\alpha(t) \gg 0($ resp., $\alpha(t) \ll 0)$.

Proof. If a nontrivial $2 \pi$-periodic solution of equation (2.9) vanishes at some point $t_{0}$ then it vanishes also at $t_{0}+2 \pi$. Consider the following two cases.

Case 1. If $x(t)$ does not change sign in $\left(t_{0}, 2 \pi+t_{0}\right)$, then $x(t)$ must reach its maximum or minimum at the point $t_{0}$, hence $x\left(t_{0}\right)=x^{\prime}\left(t_{0}\right)=0$. It follows from the uniqueness of solutions of the initial value problem that $x(t) \equiv 0$, a contradiction.

Case 2. If $x(t)$ changes sign in $\left(t_{0}, 2 \pi+t_{0}\right)$, then $x(t)$ vanishes at some $t_{1}$ in $\left(t_{0}, 2 \pi+t_{0}\right)$, so the length of at least one of the intervals $\left(t_{0}, t_{1}\right)$ and $\left(t_{1}, 2 \pi+t_{0}\right)$ is less than or equal to $\pi$. Without loss of generality we assume that $\left|t_{1}-t_{0}\right| \leq \pi$. Consider the B.V.P.

$$
\left(e^{c t} x^{\prime}\right)^{\prime}+\lambda e^{c t}=0, \quad x\left(t_{0}\right)=x\left(t_{0}+\pi\right) .
$$


It is easy to verify that the $n$th eigenvalue of $(2.10) \lambda_{n}=n^{2}+\frac{c^{2}}{4}$ and $y(t)=$ $e^{-\frac{1}{2} c\left(t-t_{0}\right)} \sin \left(t-t_{0}\right)$ corresponds to the first eigenvalue $\lambda_{1}=1+\frac{c^{2}}{4}$, but $x(t)$ is a solution of the following B.V.P.:

$$
\left(e^{c t} x^{\prime}\right)^{\prime}+e^{c t} \alpha(t) x=0, x\left(t_{0}\right)=x\left(t_{1}\right) .
$$

Since $\alpha(t) \ll \lambda_{1}$, it follows from the Sturm comparison theorem that $y(t)$ has a zero in $\left(t_{0}, t_{1}\right)$, a contradiction. (2) follows by the same argument as in [8]. To prove (3), suppose that $\alpha(t) \gg 0$. Let $\alpha_{2}(t)=\alpha(t)$ and $\alpha_{1}(t)=0$. Applying conclusion (2), we have that $L_{\alpha} x=0$ has no nontrivial $2 \pi$-periodic solution. In order to compute the local index, let us consider following parametric equation:

$$
\phi_{\lambda} x=x^{\prime \prime}+c x^{\prime}+[\lambda \alpha(t)+(1-\lambda) \bar{\alpha}] x=0
$$

where $\lambda \in[0,1]$. Obviously $\lambda \alpha(t)+(1-\lambda) \bar{\alpha} \gg 0$, thus $\phi_{\lambda} x=0$ does not admit a nontrivial $2 \pi$-periodic solution. Let $B_{\varepsilon}$ be the $\varepsilon$-ball of 0 : then $\phi_{\lambda} x=0$ has no $2 \pi$ periodic solution on $\partial B_{\varepsilon}$ for all $\lambda \in[0,1]$. By the homotopy invariance properties of the topological degree, we have that

$$
\begin{aligned}
\operatorname{ind}\left(L_{\alpha}, 0\right) & =\operatorname{deg}\left(L_{\alpha}, B_{\epsilon}, 0\right)=\operatorname{deg}\left(\phi_{\lambda}, B_{\epsilon}, 0\right) \\
& =\operatorname{deg}\left(\phi_{0}, B_{\varepsilon}, 0\right)=\operatorname{sgn}\left|\begin{array}{cc}
0 & 1 \\
-\bar{\alpha} & 0
\end{array}\right|=1 .
\end{aligned}
$$

\section{Proof of Theorem 1}

This section is devoted to proving Theorem 1. We will divide the proof into several lemmas.

Define $F: C^{2}(\mathbb{R} / 2 \pi \mathbb{Z}) \rightarrow C(\mathbb{R} / 2 \pi \mathbb{Z})$ by

$$
F(x(t))=x^{\prime \prime}+c x^{\prime}+g(t, x(t))
$$


We have the following lemma.

Lemma 3.1. $F: C^{2}(\mathbb{R} / 2 \pi \mathbb{Z}) \rightarrow C(\mathbb{R} / 2 \pi \mathbb{Z})$ is proper.

Proof. In order to show the properness of $F$, we first show that if $F\left(x_{n}\right)=h_{n}$, $\left\{h_{n}\right\} \subset C(\mathbb{R} / 2 \pi \mathbb{Z})$, is bounded, then $\left\{x_{n}\right\}$ is bounded in $C(\mathbb{R} / 2 \pi \mathbb{Z})$.

Suppose on the contrary that $\left\{x_{n}\right\}$ is unbounded in $C(\mathbb{R} / 2 \pi \mathbb{Z})$. Then there is a subsequence (still denoted by $x_{n}$ ) such that $\left\|x_{n}\right\| \rightarrow \infty$, as $n \rightarrow \infty$. Let $z_{n}=x_{n} /\left\|x_{n}\right\| ;$ then $z_{n}$ satisfies

$$
z_{n}^{\prime \prime}+c z_{n}^{\prime}+g\left(t, x_{n}\right) /\left\|x_{n}\right\|=h_{n} /\left\|h_{n}\right\|
$$

$\forall \varphi \in C^{1}(\mathbb{R} / 2 \pi \mathbb{Z})$, so we have that

$$
-\int_{0}^{2 \pi} z_{n}^{\prime} \varphi^{\prime} d t+c \int_{0}^{2 \pi} z_{n}^{\prime} \varphi d t+\int_{0}^{2 \pi} \frac{g\left(t, x_{n}\right) \varphi}{\left\|x_{n}\right\|} d t=\int_{0}^{2 \pi} \frac{\varphi h_{n}}{\left\|x_{n}\right\|} d t .
$$

Since $\left\{g\left(t, x_{n}\right) /\left\|x_{n}\right\|\right\}$ is bounded, it is precompact in weak*-topology in $L^{1}[0,2 \pi]$, thus there is a sequence such that $g\left(t, x_{n}\right) /\left\|x_{n}\right\| \rightarrow w(t)$. Taking the limit in equation (3.2), one obtains that

$$
-\int_{0}^{2 \pi} z^{\prime} \varphi^{\prime} d t+c \int_{0}^{2 \pi} z^{\prime} \varphi d t+\int_{0}^{2 \pi} w z \varphi d t=0
$$

where

$$
w(t)=\lim _{n \rightarrow \infty} \frac{g\left(t, x_{n}(t)\right)}{x_{n}(t)}= \begin{cases}\beta(t) & \text { if } z(t)>0, \\ g^{\prime}(t, 0) & \text { if } z(t)=0, \\ \alpha(t) & \text { if } z(t)<0 .\end{cases}
$$

By a regularity argument, $z(t)$ is a classical solution of the following equation:

$$
z^{\prime \prime}+c z^{\prime}+w(t) z=0
$$

Since $\|z(t)\|=1$, and $w(t) \leq \max \{\alpha(t), \beta(t)\}=p(t) \ll 1+c^{2} / 4$, it follows from Lemma 2.3 that $z(t) \neq 0$ for all $t \in R$. Thus $z(t)>0$ or $z(t)<0$, hence $w(t)=\beta(t)$ 
or $w(t)=\alpha(t)$, which contradicts Lemma 2.3, and therefore $\left\{x_{n}\right\}$ is bounded in $C(\mathbb{R} / 2 \pi \mathbb{Z})$.

Next we will show that there is a subsequence of $\left\{x_{n}\right\}$ such that converges in $C^{2}$-norm.

From the periodic boundary condition, it follows that there is $\tau_{n} \in[0,2 \pi]$ such that $x^{\prime}\left(\tau_{n}\right)=0$, therefore

$$
x_{n}^{\prime}(t)=\int_{\tau_{n}}^{t} e^{-c t}\left(h_{n}-g\left(t, x_{n}(t)\right)\right) d t \quad t \in[0,2 \pi] .
$$

This implies that $x_{n}^{\prime}(t)$ is bounded, hence the differential equation (1.1) implies that $x_{n}^{\prime \prime}(t)$ is bounded in $C(\mathbb{R} / 2 \pi \mathbb{Z})$, thus $\left\{x_{n}^{\prime}(t)\right\}$ is equicontinuous. One may choose a subsequence such that $x_{n} \rightarrow x$ in $C^{1}(\mathbb{R} / 2 \pi \mathbb{Z})$. For this same sequence we have that $x_{n}^{\prime \prime} \rightarrow x^{\prime \prime}$ in $C(\mathbb{R} / 2 \pi \mathbb{Z})$, and this shows that $F$ is proper.

Lemma 3.2. Let $x_{1}$ and $x_{2}$ be two distinct $2 \pi$-periodic solutions of (1.1). Then

$$
x_{1}(t)-x_{2}(t) \neq 0 \quad \forall t \in \mathbb{R} \text {. }
$$

Proof. Let $u=x_{1}(t)-x_{2}(t)$. Then $u$ is a nontrivial $2 \pi$-periodic solution of the following equation:

$$
x^{\prime \prime}+c x^{\prime}+q(t) x=0
$$

where $q(t)=\int_{0}^{1} g^{\prime}\left(t,(1-s) x_{1}(t)+s x_{2}(t)\right) d t$. Since $q(t) \ll \max \{\alpha(t), \beta(t)\} \ll$ $1+c^{2} / 4$, the conclusion follows from Lemma 2.3.

Lemma 3.3. 0 is a regular value of $F$, and $F(x)=0$ has exactly three $2 \pi$-periodic solution.

Proof. To show that 0 is a regular value of $F$, we need to prove that any $2 \pi$-periodic solution of $F(x)=0$ is nondegenerate, since 0 is a trivial solution of $F(x)=0$, so we have to prove that the nontrivial periodic solutions are exactly two in number. 
Consider the linearized equation

$$
F^{\prime}(x) u=u^{\prime \prime}+c u^{\prime}+g^{\prime}(t, x(t))=0 .
$$

Since $g^{\prime}(t, 0) \ll 0$, Lemma 2.3-(3) implies that 0 is nondegenerate with ind $(0)=-1$, and Lemma 3.2 gives that $x(t) \neq 0 \forall t \in \mathbb{R}$ if $x(t)$ is a nontrivial periodic solution of $F(x)=0$. Since $x g^{\prime \prime}(t, x)>0(x \neq 0)$ we have

$$
g(t, x(t)) / x(t)<g^{\prime}(t, x(t)) \ll p(t) \ll 1+c^{2} / 4 .
$$

Let $q_{1}=g(t, x(t)) / x(t)$ and $q_{2}(t)=g^{\prime}(t, x(t))$. Then Lemma 2.3-(2) implies that $x(t)$ is a nondegenerate $2 \pi$-periodic solution. In order to compute the index of $x(t)$ consider the following parametric equation:

$$
L_{\lambda} u=u^{\prime \prime}+c u^{\prime}+\left[\lambda g^{\prime}(t, x(t))+(1-\lambda)\right] u=0 \quad \forall \lambda \in[0,1] .
$$

The bound in (3.7) implies that equation (3.8) does not admit a nontrivial $2 \pi$ periodic solution, $\forall \lambda \in[0,1]$. By Lemma 2.3-(3) and the homotopy invariance properties of the degree, we have that

$$
\operatorname{ind}(x(t))=\operatorname{ind}(F, x(t))=\operatorname{deg}\left(F^{\prime}, B_{\varepsilon}, 0\right)=\operatorname{deg}\left(L_{0}, B_{\varepsilon}, 0\right)=1 .
$$

Next we show that $\operatorname{deg}\left(F, B_{\varepsilon}, 0\right)=1$, for sufficiently large $R$.

Consider the following parametric equation:

$$
\phi_{\lambda}(x)=x^{\prime \prime}+c x^{\prime}+(1-\lambda) g(t, x)+\lambda p(t) x=0 .
$$

We claim that there is $R>0$ such that for $\lambda \in[0,1], \phi_{\lambda}(x)=0$ has no solution on $\partial B_{R}$. If not, let $x_{n} \in C^{2}(\mathbb{R} / 2 \pi \mathbb{Z})$ be a sequence such that $\left\|x_{n}\right\| \rightarrow \infty$, and $\phi_{\lambda_{n}}\left(x_{n}\right)=0, \lambda_{n} \in[0,1]$. Denote $x_{n} /\left\|x_{n}\right\|$ by $z_{n}$. By the same argument as in Lemma 3.1 we obtain that there is a subsequence such that $z_{n} \rightarrow z$ and $\lambda_{n} \rightarrow \lambda$. Passing to the limit, we have that

$$
z^{\prime \prime}+c z^{\prime}+q(t) z=0
$$


where

$$
q(t)= \begin{cases}(1-\lambda) \beta(t)+\lambda p(t) & \text { if } z(t)>0 \\ (1-\lambda) g^{\prime}(t, 0)+\lambda p(t) & \text { if } z(t)=0 \\ (1-\lambda) \alpha(t)+\lambda p(t) & \text { if } z(t)<0\end{cases}
$$

Since $q(t)<(1-\lambda) p(t)+\lambda p(t) \ll 1+c^{2} / 4$, and $\|z\|=1$, by Lemma 2.3-(1), we have that $z(t) \neq 0, \forall t \in \mathbb{R}$. So we can suppose that $z(t)>0$, and then $q(t)=$ $(1-\lambda) \beta(t)+\lambda p(t)$. Again by Lemma 2.3-(3) we get that $z(t) \equiv 0$, a contradiction. On the other hand, if $q(t) \gg 0$, Lemma 2.3-(3) implies that $\operatorname{deg}\left(\phi_{1}, B_{R}, 0\right)=1$. By applying the homotopy invariance property, we have that

$$
\operatorname{deg}\left(\phi_{0}, B_{R}, 0\right)=\operatorname{deg}\left(\phi_{1}, B_{R}, 0\right)=1
$$

Finally, let $k$ be the number of nontrivial $2 \pi$-periodic solutions of $F(x)=0$. Since $F$ is proper, and 0 is a regular value of $F, k$ must be finite. By the index formula, we have that

$$
1=\operatorname{deg}\left(F, B_{R}, 0\right)=\operatorname{ind}(F, 0)+\sum_{x \neq 0} \operatorname{ind}\left(F, x_{i}\right)=-1+k,
$$

hence $k=2$, namely $F(x)=0$ has exactly three $2 \pi$-periodic solutions.

Now we finish the proof of Theorem 1 . Let $[h]$ be the cardinal number of the solutions of $F(x)=h(t)$, and $\Sigma$ be the set of critical points of $F$. It follows from the result in $[3]$ that $[h]$ is finite and constant on each connected component of $C(\mathbb{R} / 2 \pi \mathbb{Z}) / F(\Sigma)$. Therefore $F(x)=h$ has exactly three $2 \pi$-periodic solutions in the component containing 0 . In particular there is an $r>0$ such that if $\|h(t)\|<r$, the number of solutions of $F(x)=h$ is three. Since topological degree and index are invariant under small perturbations of $h(t)$, Lemma 2.2 implies that one of the $2 \pi$-periodic solutions with negative index is unstable and the other two are asymptotically stable provided that $c>0$ and $g^{\prime}(t, x) \leq \frac{1}{4}\left(1+c^{2}\right)$. 


\section{NeCESSARY AND SUFFICIENT CONDITION FOR UNIQUENESS}

Proof of Theorem 3.

First, it is evident that the condition $\bar{h} \in g(\mathbb{R})$ is met: in fact by integrating equation (1.2) we have that

$$
2 \pi g(x(\tau))=\int_{0}^{2 \pi} g(x(s)) d s=\int_{0}^{2 \pi}\left[x^{\prime \prime}+f(x(s)) x^{\prime}(s)+g(x(s))\right] d s=\int_{0}^{2 \pi} h(s) d s
$$

for some $\tau \in[0,2 \pi]$, therefore $\bar{h} \in g(\mathbb{R})$.

In order to show that the condition is sufficient, we divide the proof into two steps.

Step 1: Existence. In applying the continuation theorem to obtain an existence result, we need to estimate bounds of the periodic solution. Since equation (1.2) is equivalent to the planar system $(2.3)$, let $(x(t), y(t))$ be a solution of the following parametric equation:

$$
\begin{aligned}
& x^{\prime}=y-F(x), \\
& y^{\prime}=\lambda h(t)+(1-\lambda) \bar{h}-g(x) .
\end{aligned}
$$

Then $x(t)$ satisfies

$$
x^{\prime \prime}+f(x) x^{\prime}+g(x)=\lambda h(t)+(1-\lambda) \bar{h} .
$$

Let $m=\min h(t)$ and $M=\max h(t)$. Since $x(t) \in C^{2}(\mathbb{R} / 2 \pi \mathbb{Z}), x(t)$ reaches its maximum and minimum on $[0,2 \pi]$, let $s$ be its maximum point. Then

$$
g(x(s)) \geq \lambda h(s)+(1-\lambda) \bar{h} \geq m
$$

Since $g$ is a decreasing function, it follows that $x(s) \leq g^{-1}(m)$, and similarly $\min x(t) \geq g^{-1}(M)$, so we conclude that $x(t)$ is uniformly bounded. From (4.1) it is easy to prove that $y(t)$ is uniformly bounded. Therefore there are constants $r_{1}$ 
and $r_{2}$ such that

$$
\|x(t)\|<r_{1} \quad \text { and } \quad\|y(t)\|<r_{2} .
$$

Let $\Omega=\left\{(x, y) \mid\|x\|<r_{1},\|y\|<r_{2}\right\}$ : then (4.1) has no $2 \pi$-periodic solution on $\partial \Omega, \forall \lambda \in[0,1]$, and

$$
\operatorname{deg}(G, \Omega, 0)=\operatorname{sgn}\left|\begin{array}{ll}
-f(x) & 1 \\
-g^{\prime}(x) & 0
\end{array}\right| \begin{aligned}
& (x(\tau), F(x(\tau)) \\
& =-1
\end{aligned}
$$

where $x(\tau)=g^{-1}(\bar{h})$. It follows from Lemma 2.1 that equation (1.2) has at least one $2 \pi$-periodic solution.

Step 2: Uniqueness. Since equation (1.2) is equivalent to system $(4.1)_{1}$, therefore it is sufficient to show that $(4.1)_{1}$ has a unique solution. Let $\left(x_{1}(t), y_{1}(t)\right)$ and $\left(x_{2}(t), y_{2}(t)\right)$ be two distinct solutions of $(4.1)_{1}$, and let $u(t)=y_{1}-y_{2}$ and $v(t)=$ $x_{1}-x_{2}$. Then

$$
\begin{aligned}
& v^{\prime}=u-\left(F\left(x_{1}\right)-F\left(x_{2}\right)\right), \\
& u^{\prime}=-\left(g\left(x_{1}\right)-g\left(x_{2}\right)\right) .
\end{aligned}
$$

First let us show that $u(t) \leq 0$ for $t \in[0,2 \pi]$. If not, let $s$ be the maximum point of $u(t)$. Then $u(s)>0, u^{\prime}(s)=0$ and $u^{\prime \prime}(s) \leq 0$. Since $g$ is a decreasing function, it follows from the second equation of (4.3) that $x_{1}(s)=x_{2}(s)$, therefore the first equation of (4.3) implies that

$$
0 \geq u^{\prime \prime}(s)=-\left.\frac{d}{d t}\left(g\left(x_{1}\right)-g\left(x_{2}\right)\right)\right|_{t=s}=-g^{\prime}\left(x_{1}(s)\right) u(s)>0,
$$

a contradiction. If we reverse the order of $y_{1}$ and $y_{2}$ the same argument implies that $u(t) \geq 0$. Therefore $u(t) \equiv 0$ and the second equation of $(4.3)$ gives $v(t) \equiv 0$. So we have completed the proof of Theorem 3 .

Corollary. Suppose that $f$ and $g \in C^{1}(\mathbb{R})$, and assume that $g^{\prime}(x)<0$ for $x \in \mathbb{R}$ and $\lim _{x \rightarrow \pm \infty} g(x)=\mp \infty$. Then $\forall h(t) \in C(\mathbb{R} / 2 \pi \mathbb{Z})$ the equation (1.2) has a unique $2 \pi$-periodic solution. 
Example. Consider the following differential equation:

$$
x^{\prime \prime}+f(x) x^{\prime}-\arctan x=a(1-\sin x) .
$$

Since $g(\mathbb{R})=\left(-\frac{\pi}{2}, \frac{\pi}{2}\right)$, it follows from Theorem 3 that the equation has a unique solution if and only if $a \in\left(-\frac{\pi}{2}, \frac{\pi}{2}\right)$.

\section{REFERENCES}

1. A. Ambrosetti, G. Prodi, A Primer of Nonlinear Analysis, Cambridge Studies in Advanced Mathematics, vol. 34, Cambridge University Press, Cambridge, 1993.

2. A. Castro, A.C. Lazer, Critical point theory and the number of solutions of a nonlinear Dirichlet problem, Ann. Mat. Pura Appl. (4) 120 (1979) 113-137.

3. S.N. Chow, J.K. Hale, Methods of Bifurcation Theory, Grundlehren der Mathematischen Wissenschaften, vol. 251, Springer-Verlag, New York-Berlin, 1982.

4. C. Fabry, J. Mawhin, M.N. Nkashama, A multiplicity result for periodic solutions of forced nonlinear second order ordinary differential equations, Bull. London Math. Soc. 18 (1986) $173-180$.

5. G. Katriel, Uniqueness of periodic solutions for asymptotically linear Duffing equations with strong forcing, Topol. Methods Nonlinear Anal. 12 (1998) 263-274.

6. A.C. Lazer, P.J. McKenna, On the existence of stable periodic solutions of differential equations of Duffing type, Proc. Amer. Math. Soc. 110 (1990) 125-133.

7. J. Mawhin, Topological degree and boundary value problems for nonlinear differential equations, in: M. Furi, P. Zecca (Eds.), Topological Methods for Ordinary Differential Equations, Lectures given at the First C.I.M.E. Session held in Montecatini Terme, 1991, Lecture Notes in Mathematics, vol. 1537, Springer-Verlag, Berlin, 1993, pp. 74-142.

8. R. Ortega, Stability and index of periodic solutions of an equation of Duffing type, Boll. Un. Mat. Ital. B (7) 3 (1989) 533-546.

9. G. Tarantello, On the number of solutions for the forced pendulum equation, J. Differential Equations 80 (1989) 79-93.

10. A. Zitan, R. Ortega, Existence of asymptotically stable periodic solutions of a forced equation of Lienard type, Nonlinear Analysis TMA 22 (8) (1994) 993-1003. 